\title{
Primary Cardiac Osteosarcoma in a Young Man with Severe Congestive Heart Failure
}

\author{
Ichiro Takeuchi ${ }^{1}$, Takeo Kawaguchi ${ }^{1}$, Yuichiro Kimura ${ }^{1}$, Jisho Kojima ${ }^{1}$, \\ Hiromasa Shimamura ${ }^{1}$, Noritaka Shimizu ${ }^{1}$ and Tohru Izumi ${ }^{2}$
}

\begin{abstract}
A primary cardiac malignant tumor is very rare; its prevalence is only $0.002-0.28 \%$. Among most malignant tumors, angiosarcoma, leiomyosarcoma, and mesothelioma occupy the majority. A cardiac osteosarcoma is extremely rare: to our knowledge, only 36 cases have been reported worldwide. We present a 22-year-old case featuring severe congestive heart failure. Hemodynamically the tumor led to significant obstruction of the mitral valve. The patient underwent an emergency resection operation, but multiple metastases occurred. Though the characteristics still remain unclear because of the low prevalence, it is very important that these tumors be distinguished from benign tumors because of early resection operation.
\end{abstract}

Key words: malignancy, cancer, heart failure, resection operation

(DOI: 10.2169/internalmedicine.46.6267)

\begin{abstract}
Introduction
A primary malignant cardiac tumor is very rare. In one autopsy series, the prevalence of primary cardiac malignancies was $0.002-0.28 \%(1,2)$. Most cardiac tumors are metastatic tumors, which are 20-40 times more than primary tumors (3). Myxoma is the most common among the benign ones. Among the malignant tumors, angiosarcoma, leiomyosarcoma, mesothelioma and fibrosarcoma occupy the majority, the total of which is over $75 \%$ (4). A primary cardiac osteosarcoma is extremely rare. Its radiographic characteristics are unclear, and to distinguish it from benign tumors in the early stage is difficult. A resection operation is required in the early stage, and the effectiveness of additional chemotherapy or radiation therapy is not clear. We present a 22year-old Japanese man with primary osteosarcoma featuring severe congestive heart failure. We resected the tumor in an emergency operation, which occupied the left atrium and widely infiltrated the atrial wall. But a few months later, multiple metastases occurred and he died 6 months after the surgery.
\end{abstract}

Case Report
A 22-year-old man was referred to our hospital because
of shortness of breath. His dyspnea had started 1 month
prior to the admission; it worsened and was accompanied by
orthopnea. Upon physical examination, his blood pressure
was $92 / 64$ mmHg, the pulse regular with a rate of $124 /$ min,
and his breathing rate was $32 /$ min. A diastolic rumble at the
left parasternal area and a coarse crackle in both sides were
noted. Chest radiography revealed severe bilateral pulmo-
nary congestion and cardiomegaly (CTR $62 \%$ ). Arterial
blood gas analysis showed a pH of 7.42 , PO ${ }_{2}$ of 90 mmHg,
PCO of 39 mmHg, and oxygen saturation of $94 \%$ under a
reserver O2 mask.
Transthoracic echocardiography demonstrated an abnor-
mal mass with a focal high density area the diameter of
which was 5.5 cm occupying the left atrium. This mass
seemed to be broadly attached to the atrial septum without a
stalk, and it was close to the mitral orifice. It could hemo-
dynamically cause significant obstruction (Fig. 1a). CT re-
vealed the mass with focal calcification (Fig. $1 \mathrm{~b}$ ). The pa-
tient underwent emergency surgery due to severe congestive
heart failure. Macroscopically, the resected tumor was

${ }^{1}$ Department of Cardiology, Odawara Municipal Hospital, Odawara and ${ }^{2}$ Department of Cardiology, Kitasato University School of Medicine, Sagamihara

Received for publication September 26, 2006; Accepted for publication January 22, 2007

Correspondence to Dr. Ichiro Takeuchi, takeuchi@aqr.calmnet.ne.jp 

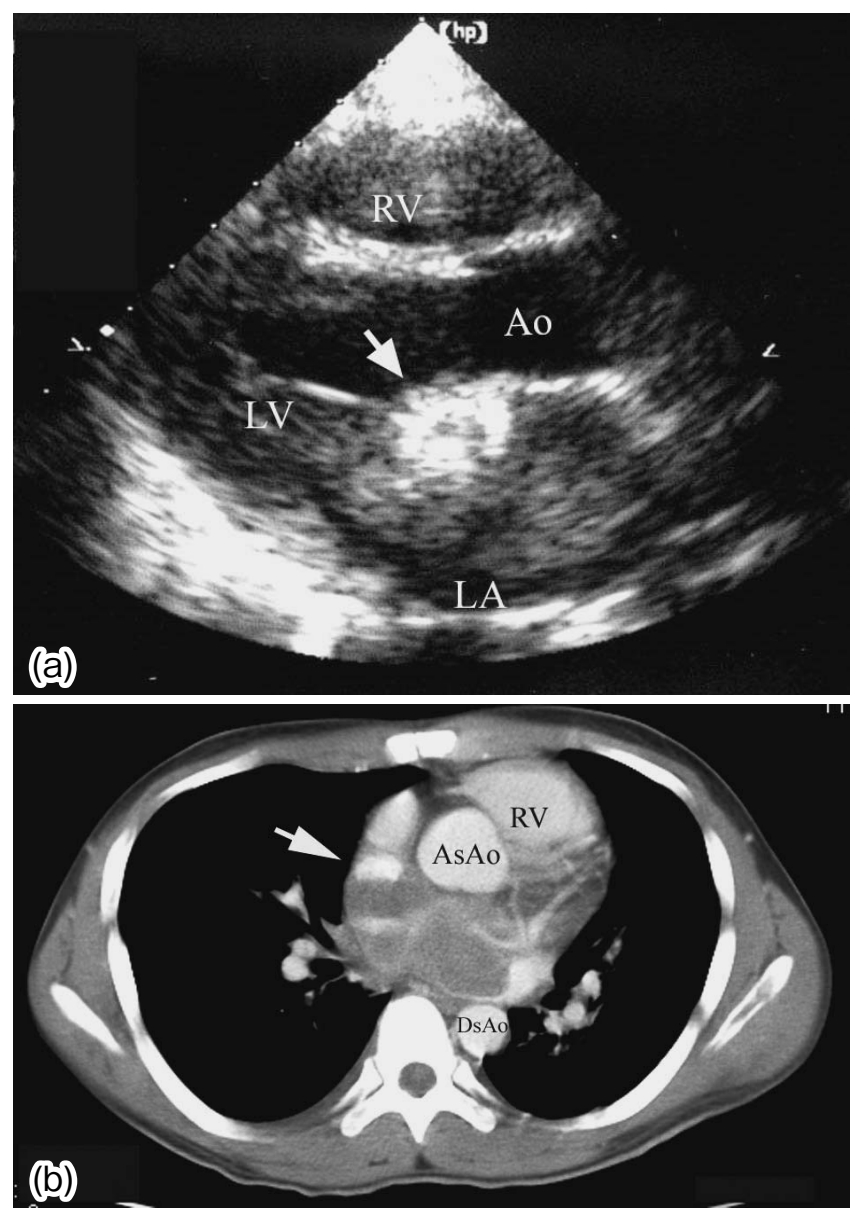

Figure 1. Echocardiography (a) and CT (b) revealed the giant tumor occupying the left atrium with focal high density pattern (arrow) without a stalk. It was attached to the atrial septum and posterior wall with invasion into the pulmonary vein. $(\mathrm{RV}=$ right ventricle; $\mathbf{A o}=$ aorta $;$ AsAo $=$ ascending aorta; DeAo = descending aorta; $L A=$ left atrium; $L V=$ left ventricle).

yellowish-gray, and elastic hard with a rough surface; it projected into the right upper pulmonary vein. It almost totally occupied the left atrium and widely infiltrated the atrial wall including the atrial septum and posterior wall, but not the mitral valve. The excised mass was $6.5 \times 5 \times 4 \mathrm{~cm}$ and weighed 70 grams (Fig. 2). Upon slicing, focal calcification was revealed accompanied by focal hemorrhaging.

Microscopically, the tumor's nuclear density was high, and mitotic figures were frequently observed. Osteoid deposits with osteoblasts and focal calcium deposits were present. So, the pathological examination demonstrated it to be a cardiac osteosarcoma (Fig. 3).

Radiographic examinations such as brain and body computed tomography (CT) did not demonstrate any other primary lesion. Following an uncomplicated postoperative course, he was discharged on the 20 postoperative day without dyspnea or pain.

Two months after the operation, his condition worsened and he experienced headaches and nausea. CT revealed two masses in his brain (diameter; $4 \mathrm{~cm}$ and $2 \mathrm{~cm}$ ) which not been previously present. He underwent another resection op-

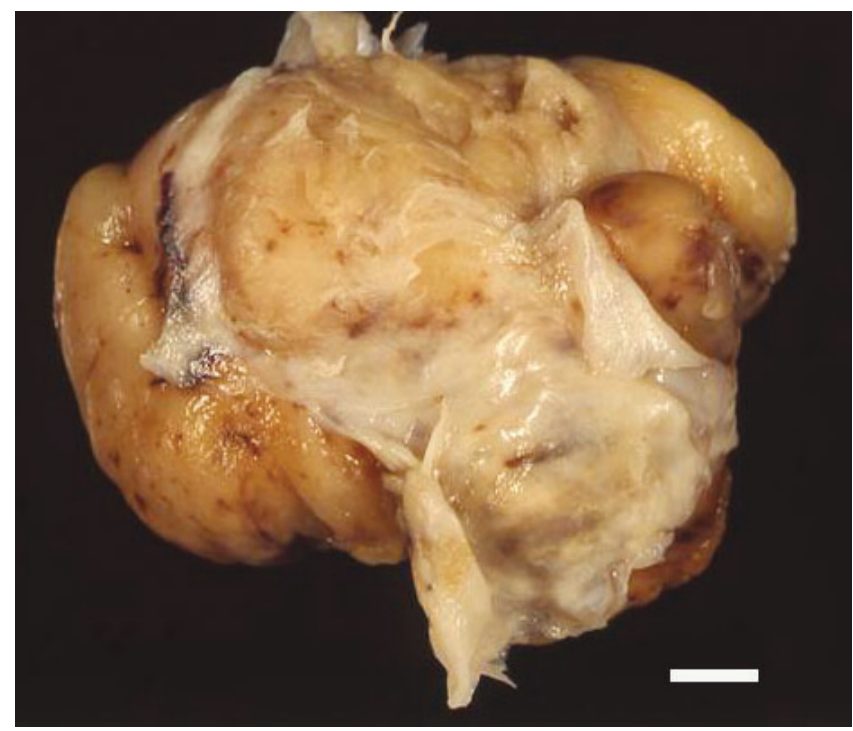

Figure 2. The resected tumor was $6.5 \times 5 \times 4 \mathrm{~cm}$ and weighed 70 grams. The bar indicates $1 \mathrm{~cm}$.

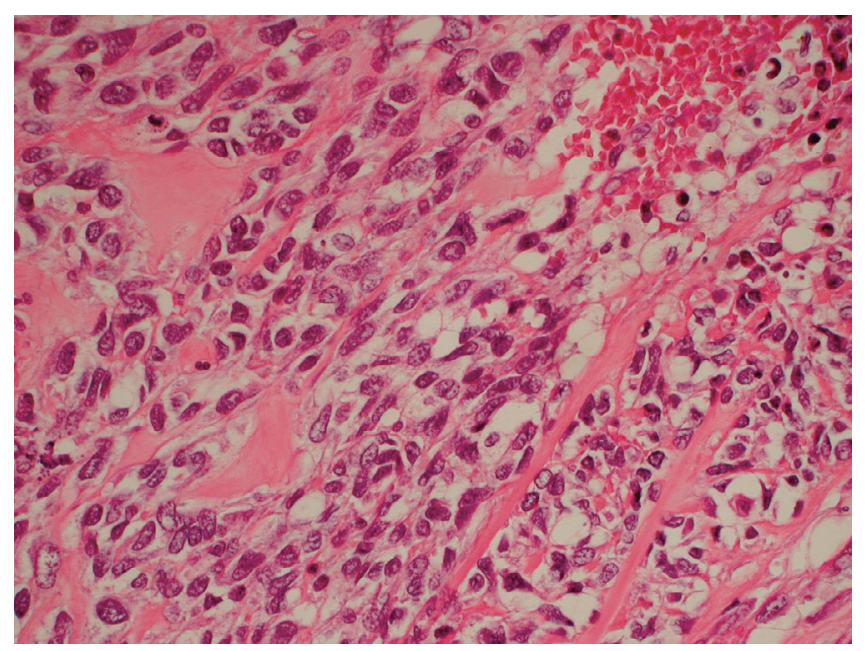

Figure 3. Histopathologic appearance of the resected tumor showed atypical spindle cells and osteoid structures with focal calcium deposits. (HE staining, $\times \mathbf{1 0 0}$ )

eration for the brain tumors. Histological analysis revealed the same tissue type and confirmed they were distant metastasis from the heart.

Four months after the first surgery, he suddenly presented with left hemiparesis.

Magnetic resonance imaging (MRI) revealed multiple metastases to the vertebral bones which compressed the spinal cord, and also to the left femur, brain, both kidneys, adrenal medulla and the left atrium. Radiation therapy was performed to the vertebral bones for suppression of local invasion. In spite of this treatment, his general condition gradually deteriorated, and he died 6 months after the initial surgery.

\section{Discussion}

Most cardiac tumors are metastatic tumors from malig- 
nant melanoma, lung carcinoma, and breast carcinoma (5). Metastases to the heart are 20-40 times more common than primary tumors (3). Primary cardiac malignant tumors are very rare. In one autopsy series, the prevalence was 0.002 $0.028 \%(1,2)$, and $75 \%$ of these were benign tumors. Myxomas at $33 \%$ are the most common among the benign tumors (4-6). Among the malignant cardiac tumors angiosarcoma is the most common, at $31.2 \%$, then leiomyosarcoma, at $20.8 \%$, mesothelioma, $15.2 \%$, fibrosarcoma, $11.2 \%$, and malignant lymphoma, at $5.6 \%(4,7)$. A primary cardiac osteosarcoma is very rare; to our knowledge only 36 cases have been reported worldwide $(4,8-22)$. In particular, malignant tumors are very rare in young people; in this case the patient was 22 years old with very rapid metastases to multiple organs.

Cardiac osteosarcoma is very rare, but the radiographic characteristics of the disease sill remain unclear. It is very important that they be distinguished from benign tumors such as myxomas because of the early resection operation. If we know beforehand whether the tumor is malignant, then a resection operation is necessary as soon as possible. The tumor should be removed as well as a wide neighboring margin and the atrial septal wall should be reconstructed with a patch. Typically, myxomas have a short broad-based attachment to the atrial septum and are pedunculated, soft, and gelatinuous. Usually they have no calcification. In this case, the high focal density area, both on CT and echocardiography, and the origin in the left atrium which broadly attached to both the atrial septum and posterior wall without a stalk, as well as invasion to the pulmonary vein, were characteristics of primary cardiac osteosarcoma. Histological examination revealed a deposit of calcium which conformed with the osteoid system, and it revealed the high density area.

The prognosis of cardiac osteosarcoma is very poor (3). This may be related to the difficulty in completely resecting the tumor and the proximity of the tumor to vital structures. In the present case, the tumor was resected in the early stage without metastasis to other organs, but a few months later, multiple metastases occurred. So patients with cardiac osteosarcoma should have frequent examinations such as CT and MRI. A resection operation is the best way, and radiation therapy and chemotherapy are not very effective. Isayama et al reported a case treated with chemotherapy under percutaneous transluminal coronary injection, but this did not improve the prognosis (13). In the present case radiation therapy was performed to suppress the local invasion. Though it could suppress the progression of the hemiparesis, the tumor had spread to the brain, adrenal medulla, femur, kidneys, and left atrium, and he died 6 months postoperatively.

\section{References}

1. McCallister HA. Primary tumors of the heart and pericardium. Curr Probl Cardiol 4: 1-51, 1979.

2. Straus R, Merliss R. Primary tumor of the heart. Arch Pathol Lab Med 39: 74-90, 1945.

3. Burke A, Virmani R. Tumors of the heart and great vessels. in: Atlas of Tumor Pathology. Fasc 16, ser3. Armed Forced Institute of Pathology, Washington DC 1996: 124-140

4. McCallister HA, Fenoglio JJ. Tumors of the cardiovascular system. in: Atlas of Tumor Pathology, series 2. Armed Forces Institute of Pathology, Washington DC 1978: 72-96

5. Hanfling SM. Metastatic cancer to the heart. Circulation 22: 474483, 1960.

6. Vander Salm TJ. Unusual primary tumor of the heart. Smin Thorac Cardiovasc Surg 12: 89-100, 2000.

7. Putnam JB, Sweeney MS, Colon R, Lanza A, Frazier OH, Cooley DA. Primary cardiac sarcomas. Ann Thorac Surg 51: 906-910, 1991.

8. Stemmerman GN, Kim PM, Berk ME. Malignant mesenchymoma of the heart. Lancet 85: 213-217, 1965.

9. Dorney P. Osteosarcoma of the heart. Br Heart J 29: 276-278, 1967.

10. Marvasti MA, Bove EL, Obeid AI, Bowser MA, Parker FB. Primary osteosarcoma of left atrium: complete surgical excision. Ann Thorac Surg 40: 402-404, 1985.

11. Dein JR, Frist WH, Stinson EB, et al. Primary cardiac neoplasms. Early and late results of surgical treatment in 42 patients. J Thorac Cardiovasc Surg 93: 502-511, 1987.

12. Burke AP, Cowan D, Virmani R. Primary sarcoma of the heart.
Cancer 69: 387-395, 1992.

13. Isayama $H$, Takeuchi $H$, Kasao M. A case of primary cardiac osteosarcoma treated with percutaneous transluminal coronary chemotherapy. Shinzo 27: 843-848, 1995.

14. Dan S, Hodge AJ. Osteogenic sarcoma of the left atrium. Ann Thorac Surg 63: 1766-1768, 1997.

15. Zanella M, Falconieri G, Bussani R. Polypoid sarcoma of the left atrium. Ann Diag Pathol 2: 167-172, 1998.

16. Jahns R, Kenn W, Stolte M. A primary osteosarcoma of the heart as a cause of recurrent peripheral arterial emboli. Ann Oncol 9: 775-778, 1998.

17. Minami H, Wakita N, Kawanishi Y, Kitano I, Sakata M, Shida T. Primary osteosarcoma of heart with severe congestive heart failure. Jpn J Thorac Cardiovasc Surg 48: 607-609, 2000.

18. Nowrangi SK, Ammash NM, Edwards WD, Breen JF, Edmonson JH. Calcified left ventricular mass: unusual clinical, echocardiographic, and computed tomographic findings of primary cardiac osteosarcoma. Mayo Clin Proc 75: 743-747, 2000.

19. Lurito KJ, Martin T, Cordes T. Right atrial primary cardiac osteosarcoma. Pediatr Cardiol 23: 462-465, 2002.

20. Kubota H, Takamoto S, Kotsuka Y, et al. Surgical treatment of malignant tumors of the right heart. Jpn Heart J 43: 263-271, 2002.

21. Shafique QS, Rajesh CM, Rajesh SN. Cardiac metastases of osteosarcoma. J Coll Physicians Surg Pak 14: 430-432, 2004.

22. Kocak H, Karapolat S, Gundogdu C, Bozkurt E, Unlu Y. Primary cardiac osteosarcoma in a pregnant woman. Heart Vessels 21: 56$58,2006$.

(C) 2007 The Japanese Society of Internal Medicine http://www.naika.or.jp/imindex.html 